\title{
An Interior-Point Solver for Optimal Power Flow Problem Considering Distributed FACTS Devices
}

\author{
Bo Liu, Lawryn Edmonds, Hang Zhang, Hongyu Wu \\ The Mike Wiegers Department of Electrical and Computer Engineering \\ Kansas State University \\ Manhattan, United States 66506
}

\begin{abstract}
In this paper, we propose an AC optimal power flow (ACOPF) model considering distributed flexible AC transmission system (D-FACTS) devices, in which the reactance of D-FACTS equipped lines are introduced as decision variables. This is motivated by increasing interests in using D-FACTS devices to address system operational and cyber-security concerns. First, DFACTS devices can be incorporated in real-time operations for economic benefits such as managing power congestions and reducing system losses. Second, D-FACTS devices can be utilized by moving target defense (MTD), an emerging concept against cyber-attacks, to prevent attackers from knowing true system configurations. Therefore, system operators can use the proposed ACOPF model to achieve economic benefits and provide the setpoints of D-FACTS devices for MTD at the same time. In addition, we rigorously derive the gradient and Hessian matrices of the objective function and constraints, which are further used to build an interior-point solver of the proposed ACOPF. Numerical results on the IEEE 118-bus transmission system show the validity of the proposed ACOPF model as well as the efficacy of the interior-point solver in minimizing system losses and generation costs.
\end{abstract}

Index Terms-ACOPF, D-FACTS device, Interior-point solver, moving target defense.

\section{INTRODUCTION}

The integration of distributed energy resources (DERs) and high penetration of DERs challenge power grids with a series of adverse effects, including line flow, reliability, and power quality issues [1]. Recently, distributed flexible AC transmission system (D-FACTS) devices have been designed and applied in power systems to enhance system stability and improve the power quality [2]. As the distributed version of conventional lumped FACTS devices, D-FACTS devices overcome the drawbacks of FACTS devices, such as bulky size, low reliability and high cost. In addition, scalable, light-weight and cost-effective D-FACTS devices can provide a variety of advanced functions and solve operational problems in the smart grid such as voltage sags, voltage fluctuations, and harmonics [3]. Therefore, D-FACTS devices are gaining recent traction and have been deployed in real-world power grids.

In addition to the benefits in power system operation, DFACTS devices are also used in moving target defense (MTD) strategies for enhancing cyber-security in modern power grids [4]-[6]. MTD actively changes the setpoints of D-FACTS devices to prevent attackers from knowing the true system configurations. It has been proven that the time-varying system configurations stemming from MTD can enable system operators to detect false data injection (FDI) attacks against the power system state estimation [4]-[6].

Since D-FACTS devices can be used to control the line impedance dynamically, system operators can utilize DFACTS devices to effectively control power flows and system power losses in real-time operations. To fully utilize D-FACTS devices, integration of D-FACTS device into the mathematical model of real-time operations is necessary. As significant tools in real-time power system operation and control, optimal power flow (OPF) models can determine the minimum operating cost and system losses, as well as retain the control variables in secure boundaries. In the literature, a rectangular representation of FACTS devices such as Phase Shift Transformer and the Unified Power Flow Controller were integrated into AC optimal power flow (ACOPF) [7]. Work also has been done on the incorporation of D-FACTS devices in DC optimal power flow (DCOPF) to study the impact of MTD on system costs [6]. However, the DCOPF model cannot be used to minimize system losses, which is one of the main functions of D-FACTS devices.

Although AC power flow models are widely used in practical power systems, ACOPF with the model of D-FACTS devices is still missing in the literature. To fill this gap, we propose an ACOPF model considering the D-FACTS devices, in which the reactance of lines equipped with D-FACTS devices are introduced as decision variables. The proposed ACOPF model can be applied in the control center to achieve the minimum system losses and generation costs in real-time while determining the setpoints of D-FACTS devices in MTD simultaneously. Note that an important reason for the existence of this gap is that the development of an efficient solver to solve the ACOPF model considering D-FACTS devices remains challenging, as the impedance variables introduced substantially complicate the solution of the ACOPF problem. Even though intelligent computational algorithms, such as particle swarm optimization (PSO), genetic algorithm (GA), simulated annealing (SA), and differential evolution (DE), can be used to resolve ACOPF considering the FACTS device without deriving the gradient and Hessian matrices [8], low computational efficiency of these algorithms exclude themselves to be used in real-time. On the other hand, it has been proven that interior-point methods are efficient tools to resolve the ACOPF problem [9], [10]. Therefore, we first derive the gradient and Hessian matrices of the objective function and the constraints in the proposed ACOPF model with respect to branch impedance. Then, we develop an 
interior-point solver to resolve the proposed ACOPF by modifying and extending Matlab Interior-Point Solver (MIPS) in MATPOWER developed for the conventional ACOPF [11].

It is worth mentioning that there are mainly three types of D-FACTS devices, namely, distributed series static compensator (DSSC), distributed series reactor (DSR), and distributed series impedance (DSI). DSR and DSI are designed to adjust the impedance of power lines while DSSC is similar to a phase shifter. Since the ACOPF model considering phase shifter transformer has been studied in [7], this paper focuses on integrating DSR and DSI into the ACOPF model.

The rest of this paper is organized as follows. The formulation for the ACOPF model considering D-FACTS devices is proposed in Section II. Gradient and Hessian matrices for the proposed interior-point solver are rigorously derived in Section III. Case studies are conducted in Section IV. The paper is summarized and concluded in Section V.

\section{PROBLEM FORMULATION}

After the installation of D-FACTS devices on power lines, system operators can change the setpoints of D-FACTS devices to control the impedance of these lines. Since the line impedance parameters are independent variables in the steadystate power flow problem, system operators can utilize DFACTS devices to control power flows, manage power congestion, and reduce system power losses. Therefore, we introduce the reactance of lines equipped with D-FACTS devices as decision variables in the conventional ACOPF model. The proposed ACOPF model with an objective of minimizing system losses and generation costs is formulated as:

$$
\begin{aligned}
& \min _{\mathbf{X}} \omega_{1} L^{s}(\mathbf{X})+\omega_{2} \sum_{i=1}^{n_{g}} f^{i}\left(p_{g}^{i}\right) \\
& \text { s.t. } \quad L^{s}(\mathbf{X})=\sum_{i=1}^{n_{l}} S_{i}^{f}+S_{i}^{t} \\
& g_{P}\left(\boldsymbol{\theta}, \mathbf{V}, \mathbf{P}_{\mathbf{g}}, \mathbf{x}\right)=0 \\
& g_{Q}\left(\boldsymbol{\theta}, \mathbf{V}, \mathbf{Q}_{\mathbf{g}}, \mathbf{x}\right)=0 \\
& h_{f}(\boldsymbol{\theta}, \mathbf{V}, \mathbf{x}) \leq 0 \\
& h_{t}(\boldsymbol{\theta}, \mathbf{V}, \mathbf{x}) \leq 0 \\
& \theta_{\text {ref }} \leq \theta_{0} \leq \theta_{\text {ref }} \\
& v_{i}^{\min } \leq v_{i} \leq v_{i}^{\max }, \\
& i=1, \ldots, n_{b} \\
& p_{i}^{\min } \leq p_{i} \leq p_{i}^{\max }, \\
& i=1, \ldots ., n_{g} \\
& q_{i}^{\min } \leq q_{i} \leq q_{i}^{\max }, \\
& i=1, \ldots ., n_{g} \\
& \left|x_{i}-x_{i}^{0}\right| \leq \eta x_{i}^{0} \text {, }
\end{aligned}
$$

where $\mathbf{X}=\left[\begin{array}{lllll}\boldsymbol{\theta} & \mathbf{V} & \mathbf{P}_{\mathbf{g}} & \mathbf{Q}_{\mathrm{g}} & \mathbf{x}\end{array}\right]$ are decision variables corresponding to voltage angle, voltage magnitude, generator active generation, generator reactive generation, and reactance of D-FACTS lines, respectively; $\omega_{1}$ and $\omega_{2}$ are weight parameters; $n_{b}, n_{l}, n_{g}$ and $n_{D F}$ are the number of buses, lines, generators, and D-FACTS lines, respectively; $L^{s}(\mathbf{X})$ is the system loss; $f^{i}$ is the active power generation cost of the $i$-th generator; $S_{i}^{f}$ and $S_{i}^{t}$ are complex power flows at the fromend and to-end of the $i$-th line; (1b) and (1c) are nonlinear equality constraints of the nodal active and reactive power balance, respectively; (1d) and (1e) are nonlinear inequality constraints of line power flow limits corresponding to lines starting from from-end and to-end, respectively; (1f) and (1g) are voltage angle and magnitude constraints; (1h) and (1i) are generator constraints; in $(1 \mathrm{j}), \eta$ in $\%$ reflects the physical capacity of D-FACTS devices.

\section{SOLUTION METHODOLOGIES}

In this section, we utilize the interior-point solver to solve the proposed ACOPF model by modifying and extending the MIPS in MATPOWER [11]. Work in [11] provides the first derivatives and Hessian matrices of objective function and constraints in the conventional ACOPF model. More specifically, voltages are in polar coordinates and nodal balance equations are expressed by complex power. Here, we follow suit and extend the interior-point solver for the proposed ACOPF model. We derive the gradient and Hessian matrices of nonlinear equality constraints, inequality constraints, and objective function with respect to the reactance of lines equipped with D-FACTS devices.

\section{A. Preliminaries in Derivatives in ACOPF Model}

Let $\mathbf{V}$ be a vector of complex voltages of all buses. Then, the first derivatives of complex voltage with respect to voltage angle and magnitude are given as follows:

$$
\begin{gathered}
\mathbf{V}_{\boldsymbol{\theta}}=\frac{\partial \mathbf{V}}{\partial \boldsymbol{\theta}}=j[\mathbf{V}] \\
\mathbf{V}_{\mathbf{v}}=\frac{\partial \mathbf{V}}{\partial \mathbf{v}}=[\mathbf{E}]
\end{gathered}
$$

where $\boldsymbol{\theta}$ is the voltage angle vector; $\boldsymbol{v}$ is the voltage magnitude vector; $\mathbf{E}=[\mathbf{v}]^{-1} \mathbf{V} ;[\bullet]$ is a diagonalizable operator defined in [11], which converts a vector to a diagonal matrix, i.e., $[\bullet]: \mathbb{C}^{n} \rightarrow \mathbb{C}^{n \times n}$. For example, when we apply the diagonalizable operator on $\mathbf{b}=\left[\begin{array}{l}1 \\ 2 \\ 3\end{array}\right]$, we have $[\mathbf{b}]=\left[\begin{array}{lll}1 & 0 & 0 \\ 0 & 2 & 0 \\ 0 & 0 & 3\end{array}\right]$.

As independent variables in the proposed ACOPF model, the line reactance controlled by the D-FACTS devices directly determines the nodal admittance matrix. Since the nodal admittance matrix plays an important role in deriving the derivatives of objective function and constraints with respect to the reactance, we present the definition of nodal admittance in MATPOWER. MATPOWER models line parameters, transformers and shunt elements in the nodal admittance matrix, which is defined as:

$\mathbf{Y}_{\text {bus }}=C_{f}^{T}\left(\left[\mathbf{Y}_{f f}\right] C_{f}+\left[\mathbf{Y}_{f t}\right] C_{t}\right)+C_{t}^{T}\left(\left[\mathbf{Y}_{t f}\right] C_{f}+\left[\mathbf{Y}_{t t}\right] C_{t}\right)+\left[\mathbf{Y}_{s h}\right]$

where $Y_{f t}^{i}=-y_{s}^{i}\left(\tau e^{-j \theta_{s h}}\right)^{-1}, Y_{t f}^{i}=-y_{s}^{i}\left(\tau e^{j \theta_{s h}}\right)^{-1}, Y_{t t}^{i}=y_{s}^{i}+j 0.5 b_{c}$ and $Y_{f f}^{i}=\left(y_{s}^{i}+j 0.5 b_{c}\right) \tau^{-2}$ are equivalent admittance of the $i$-th line between different ends in the standard $\pi$ transmission line model; $y_{s}^{i}$ is the admittance of the $i$-th line, i.e., $y_{s}^{i}=\left(r_{i}+j x_{i}\right)^{-1}$, and $r_{i}$ and $x_{i}$ are resistance and reactance of the $i$-th line, respectively; $\tau$ is the transformer tap ratio magnitude, and $\theta_{s h}$ is the transformer phase shift angle; $Y_{s h}^{i}$ is admittance of shunt elements of the $i$-th bus; $C_{t}$ and $C_{f}$ are 
connection matrices used in building the system admittance matrices, defined in [11]. The first and second derivatives of $y_{s}^{i}$ with respect to the line reactance are calculated as:

$$
\begin{gathered}
\frac{\partial y_{s}^{i}}{\partial x_{i}}=\frac{-2 x_{i} r_{i}+j\left(x_{i}^{2}-r_{i}^{2}\right)}{\left(x_{i}^{2}+r_{i}^{2}\right)^{2}} \\
\frac{\partial^{2} y_{s}^{i}}{\partial x_{i}^{2}}=2 \frac{r\left(3 x_{i}^{2}-r_{i}^{2}\right)+j x_{i}\left(3 r_{i}^{2}-x_{i}^{2}\right)}{\left(x_{i}^{2}+r_{i}^{2}\right)^{3}}
\end{gathered}
$$

The gradient of $\mathbf{Y}_{f f}, \mathbf{Y}_{f t}, \mathbf{Y}_{t f}$ and $\mathbf{Y}_{t t}$ with respect to line reactance are diagonal matrices and their diagonal entries can be calculated as follows:

$$
\begin{gathered}
\nabla_{\mathbf{x}} \mathbf{Y}_{f f}(i, i)=\frac{\partial \mathbf{Y}_{f f}}{\partial \mathbf{x}}(i, i)=\frac{1}{\tau^{2}} \frac{\partial y_{s}^{i}}{\partial x_{i}} \\
\nabla_{\mathbf{x}} \mathbf{Y}_{f t}(i, i)=-\frac{1}{\tau e^{-j \theta_{s h}}} \frac{\partial y_{s}^{i}}{\partial x_{i}} \\
\nabla_{\mathbf{x}} \mathbf{Y}_{t f}(i, i)=-\frac{1}{\tau e^{j \theta_{s h}}} \frac{\partial y_{s}^{i}}{\partial x_{i}} \\
\nabla_{\mathbf{x}} \mathbf{Y}_{t t}(i, i)=\frac{\partial y_{s}^{i}}{\partial x_{i}}
\end{gathered}
$$

Similarly, the Hessian matrices of $\mathbf{Y}_{f f}, \mathbf{Y}_{f t}, \mathbf{Y}_{t f}, \mathbf{Y}_{t t}$ are diagonal matrices as shown in (11), whose diagonal entries can be calculated as $\nabla_{\mathbf{x x}}^{2} \mathbf{Y}_{f f}(i, i)=\frac{1}{\tau^{2}} \frac{\partial^{2} y_{s}^{i}}{\partial x_{i}^{2}}, \nabla_{\mathbf{x x}}^{2} \mathbf{Y}_{t t}(i, i)=\frac{\partial^{2} y_{s}^{i}}{\partial x_{i}^{2}}$, $\nabla_{\mathbf{x x}}^{2} \mathbf{Y}_{f t}(i, i)=-\frac{1}{\tau e^{-j \theta_{s h}}} \frac{\partial^{2} y_{s}^{i}}{\partial x_{i}^{2}}$, and $\nabla_{\mathbf{x x}}^{2} \mathbf{Y}_{t f}(i, i)=-\frac{1}{\tau e^{j \theta_{s h}}} \frac{\partial^{2} y_{s}^{i}}{\partial x_{i}^{2}}$,

respectively. Note that the first and second derivatives of the admittance of shunt elements with respect to reactance are zero matrices, i.e., $\nabla_{\mathbf{x}} \mathbf{Y}_{s h}=\mathbf{0}$ and $\nabla_{\mathbf{x} \mathbf{x}}^{2} \mathbf{Y}_{s h}=\mathbf{0}$. For presentation simplicity, the subscripts of the gradient and Hessian matrices of all admittance matrices with respect to the reactance are omitted hereinafter, i.e., $\nabla \mathbf{Y}_{f f}=\nabla_{\mathbf{x}} \mathbf{Y}_{f f}$ and $\nabla^{2} \mathbf{Y}_{f f}=\nabla_{\mathbf{x x}}^{2} \mathbf{Y}_{f f}$.

$$
\begin{aligned}
& \nabla^{2} \mathbf{Y}_{f f}=\frac{\partial}{\partial \mathbf{x}}\left(\left(\frac{\partial}{\partial \mathbf{x}} \mathbf{Y}_{f f}^{T}\right) \times \mathbf{a}\right)= \\
& =\left[\begin{array}{cccc}
\frac{\partial}{\partial x_{1}}\left(\frac{\partial Y_{f f}^{1}}{\partial x_{1}}\right) & \frac{\partial}{\partial x_{2}}\left(\frac{\partial Y_{f f}^{1}}{\partial x_{1}}\right) & \cdots & \frac{\partial}{\partial x_{n_{l}}}\left(\frac{\partial Y_{f f}^{1}}{\partial x_{1}}\right) \\
\frac{\partial}{\partial x_{1}}\left(\frac{\partial Y_{f f}^{2}}{\partial x_{2}}\right) & \frac{\partial}{\partial x_{2}}\left(\frac{\partial Y_{f f}^{2}}{\partial x_{2}}\right) & \cdots & \frac{\partial}{\partial x_{n l}}\left(\frac{\partial Y_{f f}^{2}}{\partial x_{2}}\right) \\
\vdots & \vdots & \ddots & \vdots \\
\frac{\partial}{\partial x_{1}}\left(\frac{\partial Y_{f f}^{n}}{\partial x_{n_{l}}}\right) & \frac{\partial}{\partial x_{2}}\left(\frac{\partial Y_{f f}^{n}}{\partial x_{n_{l}}}\right) & \cdots & \frac{\partial}{\partial x_{n_{l}}}\left(\frac{\partial Y_{f f}^{n}}{\partial x_{n_{l}}}\right)
\end{array}\right]=\left[\begin{array}{cccc}
\frac{\partial^{2} Y_{f f}^{1}}{\partial x_{1}^{2}} & 0 & \cdots & 0 \\
0 & \frac{\partial^{2} Y_{f f}^{2}}{\partial x_{2}^{2}} & \cdots & 0 \\
\vdots & \vdots & \ddots & \vdots \\
0 & 0 & \cdots & \frac{\partial^{2} Y_{f f}^{n}}{\partial x_{n_{l}}^{2}}
\end{array}\right]
\end{aligned}
$$

where $\mathbf{a}=\mathbf{1} \in \mathbb{R}^{n_{l} \times 1}$.

\section{B. Gradient of Power Injection Constraints}

The complex power balance equations can be expressed as $G^{s}(\mathbf{X})=\mathbf{S}^{b u s}+\mathbf{S}_{d}-\mathbf{C}_{g} \mathbf{S}_{g}=\mathbf{0}$, where $\mathbf{S}_{d}$ is a complex power load vector of all buses; $\mathbf{S}_{g}$ is a complex power generation vector of all buses; $\mathbf{S}^{\text {bus }}$ is a complex power injection vector of all buses, i.e., $\mathbf{S}^{\text {bus }}=[\mathbf{V}] \mathbf{I}^{\text {bus }}$, and $\mathbf{I}^{\text {bus }}$ is a complex current injection vector, i.e., $\mathbf{I}^{\text {bus }}=\mathbf{Y}_{\text {bus }} \mathbf{V}$. The gradient of power balance equations can be expressed as follows:

$$
G_{\mathbf{X}}^{s}(\mathbf{X})=\left[\begin{array}{lllll}
G_{\mathbf{\theta}}^{s} & G_{\mathrm{v}}^{s} & G_{\mathbf{P}_{\mathbf{g}}}^{s} & G_{\mathbf{Q}_{\mathrm{g}}}^{s} & G_{\mathbf{x}}^{s}
\end{array}\right]
$$

where the first four items irrelative to the line reactance are consistent with the results in [11]. We can calculate $G_{\mathbf{x}}^{s}$ by using (4) and (7-10) as follows:

$G_{\mathbf{x}}^{s}=\mathbf{S}_{\mathbf{x}}^{\text {bus }}=\frac{\partial}{\partial \mathbf{x}}\left([\mathbf{V}] \mathbf{Y}_{\text {bus }}^{*} \mathbf{V}^{*}\right)$

$=\frac{\partial}{\partial \mathbf{x}}\left\{[\mathbf{V}]\left\{C_{f}^{T}\left(\left[\mathbf{Y}_{f f}\right] C_{f}+\left[\mathbf{Y}_{f t}\right] C_{t}\right)+C_{t}^{T}\left(\left[\mathbf{Y}_{t f}\right] C_{f}+\left[\mathbf{Y}_{t t}\right] C_{t}\right)+\left[\mathbf{Y}_{s h}\right]\right\} \mathbf{V}^{*}\right\}$

$=\frac{\partial}{\partial \mathbf{x}}\left\{[\mathbf{V}]\left\{C_{f}^{T}\left(\left[C_{f} \mathbf{V}^{*}\right] \mathbf{Y}_{f f}^{*}+\left[C_{t} \mathbf{V}^{*}\right] \mathbf{Y}_{f t}^{*}\right)+C_{t}^{T}\left(\left[C_{f} \mathbf{V}^{*}\right] \mathbf{Y}_{t f}^{*}+\left[C_{t} \mathbf{V}^{*}\right] \mathbf{Y}_{t t}^{*}\right)\right\}\right\}$

$=[\mathbf{V}] C_{f}^{T}\left(\left[C_{f} \mathbf{V}^{*}\right] \nabla \mathbf{Y}_{f f}^{*}+\left[C_{t} \mathbf{V}^{*}\right] \nabla \mathbf{Y}_{f t}^{*}\right)$

$+[\mathbf{V}] C_{t}^{T}\left(\left[C_{f} \mathbf{V}^{*}\right] \nabla \mathbf{Y}_{t f}^{*}+\left[C_{t} \mathbf{V}^{*}\right] \nabla \mathbf{Y}_{t t}^{*}\right)$

\section{Hessian Matrix of Power Injection Constraints}

The Hessian matrix of complex power balance constraints in the proposed ACOPF can be expressed as:

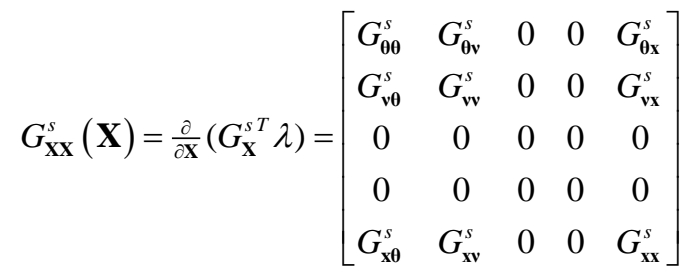

where $\lambda$ is a constant vector for calculating the Hessian matrix; the expressions of $G_{\theta \theta}^{s}, G_{\theta \mathrm{v}}^{s}, G_{\mathrm{v} \theta}^{s}$ and $G_{\mathrm{vv}}^{s}$ can be found in [11]; $G_{\mathbf{x x}}^{s}, G_{\mathbf{x v}}^{s}, G_{\mathbf{x} \theta}^{s}, G_{\mathbf{\theta x}}^{s}$, and $G_{\mathbf{v x}}^{s}$ need to be derived for the proposed ACOPF model. Due to the space limit, we ignore the derivation process and directly present the calculation results. First, we calculate $G_{\mathbf{x x}}^{s}$ using (13).

$$
\begin{aligned}
G_{\mathbf{x x}}^{s}= & \frac{\partial}{\partial \mathbf{x}}\left(G_{\mathbf{x}}^{T} \lambda\right) \\
= & {\left[C_{f}[\mathbf{V}] \lambda\right]\left(\left[C_{f} \mathbf{V}^{*}\right] \nabla_{\mathbf{x x}}^{2} \mathbf{Y}_{f f}^{*}+\left[C_{t} \mathbf{V}^{*}\right] \nabla^{2} \mathbf{Y}_{f t}^{*}\right) } \\
& +\left[C_{t}[\mathbf{V}] \lambda\right]\left(\left[C_{f} \mathbf{V}^{*}\right] \nabla_{\mathbf{x x}}^{2} \mathbf{Y}_{t f}^{*}+\left[C_{t} \mathbf{V}^{*}\right] \nabla^{2} \mathbf{Y}_{t t}^{*}\right)
\end{aligned}
$$

Similarly, we calculate $G_{\mathbf{x v}}^{s}, G_{\mathbf{v x}}^{s}, G_{\mathbf{x} \boldsymbol{\theta}}^{s}, G_{\mathbf{\theta} \mathbf{x}}^{s}$ as follows:

$$
\begin{aligned}
G_{\mathbf{x v}}^{s}= & \left(\left[\nabla \mathbf{Y}_{f f}^{*} C_{f}[\mathbf{V}] \lambda\right]+\left[\nabla \mathbf{Y}_{t f}^{*} C_{t}[\mathbf{V}] \lambda\right]\right) C_{f}[\mathbf{E}]^{*} \\
& +\left(\left[\nabla \mathbf{Y}_{f t}^{*} C_{f}[\mathbf{V}] \lambda\right]+\left[\nabla \mathbf{Y}_{t t}^{*} C_{t}[\mathbf{V}] \lambda\right]\right) C_{t}[\mathbf{E}]^{*} \\
& +\left[C_{f} \mathbf{V}^{*}\right]\left(\nabla \mathbf{Y}_{f f}^{*} C_{f}+\nabla \mathbf{Y}_{t f}^{*} C_{t}\right)[\lambda][\mathbf{E}] \\
& +\left[C_{t} \mathbf{V}^{*}\right]\left(\nabla \mathbf{Y}_{f t}^{*} C_{f}+\nabla \mathbf{Y}_{t t}^{*} C_{t}\right)[\lambda][\mathbf{E}] \\
G_{\theta \mathbf{x}}^{s}= & j[\mathbf{V}][\lambda] C_{f}^{T}\left(\left[C_{f} \mathbf{V}^{*}\right] \nabla \mathbf{Y}_{f f}^{*}+\left[C_{t} \mathbf{V}^{*}\right] \nabla \mathbf{Y}_{f t}^{*}\right) \\
& +j[\mathbf{V}][\lambda] C_{t}^{T}\left(\left[C_{f} \mathbf{V}^{*}\right] \nabla \mathbf{Y}_{t f}^{*}+\left[C_{t} \mathbf{V}^{*}\right] \nabla \mathbf{Y}_{t t}^{*}\right) \\
& -j\left[\mathbf{V}^{*}\right] C_{f}^{T}\left(\left[C_{f}[\mathbf{V}] \lambda\right] \nabla \mathbf{Y}_{f f}^{*}+\left[C_{t}[\mathbf{V}] \lambda\right] \nabla \mathbf{Y}_{t f}^{*}\right) \\
& -j\left[\mathbf{V}^{*}\right] C_{t}^{T}\left(\left[C_{f}[\mathbf{V}] \lambda\right] \nabla \mathbf{Y}_{f t}^{*}+\left[C_{t}[\mathbf{V}] \lambda\right] \nabla \mathbf{Y}_{t t}^{*}\right)
\end{aligned}
$$

$$
\begin{aligned}
& G_{\mathbf{x} \theta}^{s}= j\left\{\left[C_{f} \mathbf{V}^{*}\right]\left(\nabla \mathbf{Y}_{f f}^{*} C_{f}+\nabla \mathbf{Y}_{t f}^{*} C_{t}\right)+\left[C_{t} \mathbf{V}^{*}\right]\left(\nabla \mathbf{Y}_{f t}^{*} C_{f}+\nabla \mathbf{Y}_{t t}^{*} C_{t}\right)\right\}[\lambda][\mathbf{V}] \\
&-j\left\{\left[\nabla \mathbf{Y}_{f f}^{*} C_{f}[\mathbf{V}] \lambda\right]+\left[\nabla \mathbf{Y}_{t f}^{*} C_{t}[\mathbf{V}] \lambda\right]\right\} C_{f}[\mathbf{V}]^{*} \\
&-j\left\{\left[\nabla \mathbf{Y}_{f t}^{*} C_{f}[\mathbf{V}] \lambda\right]+\left[\nabla \mathbf{Y}_{t t}^{*} C_{t}[\mathbf{V}] \lambda\right]\right\} C_{t}[\mathbf{V}]^{*} \\
& G_{\mathbf{v x}}^{s}=[\mathbf{E}][\lambda] C_{f}^{T}\left(\left[C_{f} \mathbf{V}^{*}\right] \nabla \mathbf{Y}_{f f}^{*}+\left[C_{t} \mathbf{V}^{*}\right] \nabla \mathbf{Y}_{f t}^{*}\right) \\
&+[\mathbf{E}][\lambda] C_{t}^{T}\left(\left[C_{f} \mathbf{V}^{*}\right] \nabla \mathbf{Y}_{t f}^{*}+\left[C_{t} \mathbf{V}^{*}\right] \nabla \mathbf{Y}_{t t}^{*}\right) \\
&+\left[\mathbf{E}^{*}\right] C_{f}^{T}\left(\left[C_{f}[\mathbf{V}] \lambda\right] \nabla \mathbf{Y}_{f f}^{*}+\left[C_{t}[\mathbf{V}] \lambda\right] \nabla \mathbf{Y}_{t f}^{*}\right) \\
&+\left[\mathbf{E}^{*}\right] C_{t}^{T}\left(\left[C_{f}[\mathbf{V}] \lambda\right] \nabla \mathbf{Y}_{f t}^{*}+\left[C_{t}[\mathbf{V}] \lambda\right] \nabla \mathbf{Y}_{t t}^{*}\right)
\end{aligned}
$$




\section{Gradient of Power Flow Constraints}

In the power flow constraints, we derive the gradient and Hessian matrix for the complex power flow at the from-ends of the lines. The derivative results for the to-ends of the line can be identically calculated by replacing all $f$ sub/super-scripts with $t$. Similar to power injection constraints, the first derivatives of the power flow with respect to voltage angle, voltage magnitude, real and reactive power generation are identical to that in [11]. We only derive the first derivatives of power flow with respect to reactance as follows.

$$
\begin{aligned}
\mathbf{S}_{\mathbf{x}}^{f} & =\nabla_{\mathbf{x}} \mathbf{S}_{f}=\frac{\partial}{\partial \mathbf{x}}\left\{\left[C_{f} \mathbf{V}\right]\left(\left[\mathbf{Y}_{f f}^{*}\right] C_{f}+\left[\mathbf{Y}_{f t}^{*}\right] C_{t}\right) \mathbf{V}^{*}\right\} \\
& =\left[C_{f} \mathbf{V}\right]\left(\left[C_{f} \mathbf{V}^{*}\right] \nabla \mathbf{Y}_{f f}^{*}+\left[C_{t} \mathbf{V}^{*}\right] \nabla \mathbf{Y}_{f t}^{*}\right)
\end{aligned}
$$

\section{E. Hessian Matrix of Power Flow Constraints}

The Hessian matrix of complex power flow constraints in the proposed ACOPF has the same form as that of power flow constraints. In the Hessian matrix, $\mathbf{S}_{\boldsymbol{\theta} \theta}^{f}, \mathbf{S}_{\boldsymbol{\theta} \mathbf{v}}^{f}, \mathbf{S}_{\mathbf{v} \boldsymbol{\theta}}^{f}$, and $\mathbf{S}_{\mathbf{v v}}^{f}$ are identical to that in [11]; $\mathbf{S}_{\mathbf{x x}}^{f}, \mathbf{S}_{\mathbf{x v}}^{f}, \mathbf{S}_{\mathbf{x} \theta}^{f}, \mathbf{S}_{\mathbf{\theta x}}^{f}$ and $\mathbf{S}_{\mathbf{v x}}^{f}$ can be derived as follows, using $\mathbf{S}_{\boldsymbol{\theta}}^{f}$ and $\mathbf{S}_{\mathbf{v}}^{f}$ in [11].

$$
\begin{aligned}
& \mathbf{S}_{\mathbf{x x}}^{f}=\frac{\partial}{\partial \mathbf{x}}\left(\mathbf{S}_{\mathbf{x}}^{T} \lambda\right)=\left[C_{f} \mathbf{V}\right][\lambda]\left(\left[C_{f} \mathbf{V}^{*}\right] \nabla^{2} \mathbf{Y}_{f f}^{*}+\left[C_{t} \mathbf{V}^{*}\right] \nabla^{2} \mathbf{Y}_{f t}^{*}\right) \\
& \mathbf{S}_{\mathbf{x v}}^{f}=\nabla \mathbf{Y}_{f f}^{*}[\lambda]\left(\left[C_{f} \mathbf{V}\right] C_{f}[\mathbf{E}]^{*}+\left[C_{f} \mathbf{V}^{*}\right] C_{f}[\mathbf{E}]\right) \\
&+\nabla \mathbf{Y}_{f t}^{*}[\lambda]\left(\left[C_{f} \mathbf{V}\right] C_{t}[\mathbf{E}]^{*}+\left[C_{t} \mathbf{V}^{*}\right] C_{f}[\mathbf{E}]\right) \\
& \mathbf{S}_{\mathbf{x} \mathbf{x}}^{f}= j \nabla \mathbf{Y}_{f f}^{*}[\lambda]\left(-\left[C_{f} \mathbf{V}\right] C_{f}[\mathbf{V}]^{*}+\left[C_{f} \mathbf{V}^{*}\right] C_{f}[\mathbf{V}]\right) \\
&+j \nabla \mathbf{Y}_{f t}^{*}[\lambda]\left(-\left[C_{f} \mathbf{V}\right] C_{t}[\mathbf{V}]^{*}+\left[C_{t} \mathbf{V}^{*}\right] C_{f}[\mathbf{V}]\right) \\
& \mathbf{S}_{\mathbf{v x}}^{f}= {[\mathbf{E}] C_{f}^{T}[\lambda]\left(\left[C_{f} \mathbf{V}^{*}\right] \nabla \mathbf{Y}_{f f}^{*}+\left[C_{t} \mathbf{V}^{*}\right] \nabla \mathbf{Y}_{f t}^{*}\right) } \\
&+\left[\mathbf{E}^{*}\right]\left(C_{f}^{T}\left[C_{f} \mathbf{V}\right][\lambda] \nabla \mathbf{Y}_{f f}^{*}+C_{t}^{T}\left[C_{f} \mathbf{V}\right][\lambda] \nabla \mathbf{Y}_{f t}^{*}\right) \\
& \mathbf{S}_{\theta \mathbf{x}}^{f}= j[\mathbf{V}] C_{f}^{T}[\lambda]\left(\left[C_{f} \mathbf{V}^{*}\right] \nabla \mathbf{Y}_{f f}^{*}+\left[C_{t} \mathbf{V}^{*}\right] \nabla \mathbf{Y}_{f t}^{*}\right) \\
&-j\left[\mathbf{V}^{*}\right]\left(C_{f}^{T}\left[C_{f} \mathbf{V}\right][\lambda] \nabla \mathbf{Y}_{f f}^{*}+C_{t}^{T}\left[C_{f} \mathbf{V}\right][\lambda] \nabla \mathbf{Y}_{f t}^{*}\right)
\end{aligned}
$$

\section{F. Gradient and Hessian Matrix of System Losses}

The system complex power loss is the sum of complex power loss of each line, and the line power loss is the sum of complex power flows at the from-end and to-end of this line, as shown in (1a). The system loss can be expressed in matrix form, i.e., $L^{s}=a^{T}\left(\mathbf{S}^{f}+\mathbf{S}^{t}\right)$, where $a=\mathbf{1} \in \mathbb{R}^{n_{l} \times 1}$. Therefore, the first derivative of the system loss is $L_{\mathbf{x}}^{s}=a^{T}\left(\mathbf{S}_{\mathbf{x}}^{f}+\mathbf{S}_{\mathbf{x}}^{t}\right)$.

The Hessian matrix of the system loss has the same form as that of power injection constraints in (14). Take $L_{\mathbf{x x}}^{s}$ for example, $L_{\mathbf{x x}}^{s}$ can be calculated as follows:

$$
\begin{aligned}
L_{\mathbf{x x}}^{s} & =\frac{\partial}{\partial \mathbf{x}}\left(\left(L_{\mathbf{x}}^{s}\right)^{T}\right)=\frac{\partial}{\partial \mathbf{x}}\left(\mathbf{S}_{\mathbf{x}}^{f T} a+\mathbf{S}_{\mathbf{x}}^{t T} a\right) \\
& =\left.\frac{\partial}{\partial \mathbf{x}}\left(\mathbf{S}_{\mathbf{x}}^{f T} \lambda\right)\right|_{\lambda=a}+\left.\frac{\partial}{\partial \mathbf{x}}\left(\mathbf{S}_{\mathbf{x}}^{t T} \lambda\right)\right|_{\lambda=a} \\
& =\left.\mathbf{S}_{\mathbf{x x}}^{f}\right|_{\lambda=a}+\left.\mathbf{S}_{\mathbf{x x}}^{t}\right|_{\lambda=a}
\end{aligned}
$$

Note that we have $\frac{\partial}{\partial \mathbf{x}}\left(\mathbf{S}_{\mathbf{x}}^{f T} a\right)=\left.\mathbf{S}_{\mathbf{x x}}^{f}\right|_{\lambda=a}$ according to (21).

Similarly, the remaining none-zero matrix blocks in the Hessian matrix can be calculated as follows:

$$
\begin{aligned}
& L_{\boldsymbol{\theta} \boldsymbol{\theta}}^{s}=\frac{\partial}{\partial \boldsymbol{\theta}}\left(\left(L_{\boldsymbol{\theta}}^{s}\right)^{T}\right)=\frac{\partial}{\partial \boldsymbol{\theta}}\left(\mathbf{S}_{\boldsymbol{\theta}}^{f T} a+\mathbf{S}_{\boldsymbol{\theta}}^{t T} a\right)=\left.\mathbf{S}_{\boldsymbol{\theta} \boldsymbol{\theta}}^{f}\right|_{\lambda=a}+\left.\mathbf{S}_{\boldsymbol{\theta} \boldsymbol{\theta}}^{t}\right|_{\lambda=a} \\
& L_{\mathbf{v v}}^{s}=\frac{\partial}{\partial \mathbf{v}}\left(\left(L_{\mathbf{v}}^{s}\right)^{T}\right)=\frac{\partial}{\partial \mathbf{v}}\left(\mathbf{S}_{\mathbf{v}}^{f T} a+\mathbf{S}_{\mathbf{v}}^{t T} a\right)=\left.\mathbf{S}_{\mathbf{v v}}^{f}\right|_{\lambda=a}+\left.\mathbf{S}_{\mathbf{v v}}^{t}\right|_{\lambda=a}
\end{aligned}
$$

$$
\begin{aligned}
& L_{\mathbf{x} \boldsymbol{\theta}}^{s}=\frac{\partial}{\partial \boldsymbol{\theta}}\left(\left(L_{\mathbf{x}}^{s}\right)^{T}\right)=\frac{\partial}{\partial \boldsymbol{\theta}}\left(\mathbf{S}_{\mathbf{x}}^{f T} a+\mathbf{S}_{\mathbf{x}}^{t T} a\right)=\left.\mathbf{S}_{\mathbf{x} \boldsymbol{\theta}}^{f}\right|_{\lambda=a}+\left.\mathbf{S}_{\mathbf{x} \boldsymbol{\theta}}^{t}\right|_{\lambda=a} \\
& L_{\mathbf{x v}}^{s}=\frac{\partial}{\partial \mathrm{v}}\left(\left(L_{\mathbf{x}}^{s}\right)^{T}\right)=\frac{\partial}{\partial \mathbf{v}}\left(\mathbf{S}_{\mathbf{x}}^{f T} a+\mathbf{S}_{\mathbf{x}}^{t T} a\right)=\left.\mathbf{S}_{\mathbf{x v}}^{f}\right|_{\lambda=a}+\left.\mathbf{S}_{\mathbf{x v}}^{t}\right|_{\lambda=a} \\
& L_{\theta \mathrm{v}}^{s}=\frac{\partial}{\partial \mathrm{v}}\left(\left(L_{\theta}^{s}\right)^{T}\right)=\frac{\partial}{\partial \mathrm{v}}\left(\mathbf{S}_{\boldsymbol{\theta}}^{f T} a+\mathbf{S}_{\boldsymbol{\theta}}^{t T} a\right)=\left.\mathbf{S}_{\boldsymbol{\theta}}^{f}\right|_{\lambda=a}+\left.\mathbf{S}_{\theta \mathrm{v}}^{t}\right|_{\lambda=a} \\
& L_{\mathbf{v} \boldsymbol{\theta}}^{s}=\frac{\partial}{\partial \boldsymbol{\theta}}\left(\left(L_{\mathrm{v}}^{s}\right)^{T}\right)=\frac{\partial}{\partial \boldsymbol{\theta}}\left(\mathbf{S}_{\mathbf{v}}^{f T} a+\mathbf{S}_{\mathbf{v}}^{t T} a\right)=\left.\mathbf{S}_{\mathbf{v} \boldsymbol{\theta}}^{f}\right|_{\lambda=a}+\left.\mathbf{S}_{\mathbf{v} \boldsymbol{\theta}}^{t}\right|_{\lambda=a} \\
& L_{\boldsymbol{\theta} \mathbf{x}}^{s}=\frac{\partial}{\partial \mathbf{x}}\left(\left(L_{\boldsymbol{\theta}}^{s}\right)^{T}\right)=\frac{\partial}{\partial \mathbf{x}}\left(\mathbf{S}_{\boldsymbol{\theta}}^{f T} a+\mathbf{S}_{\boldsymbol{\theta}}^{t T} a\right)=\left.\mathbf{S}_{\mathbf{\theta x}}^{f}\right|_{\lambda=a}+\left.\mathbf{S}_{\mathbf{\theta x}}^{t}\right|_{\lambda=a} \\
& L_{\mathbf{v} \mathbf{x}}^{s}=\frac{\partial}{\partial \mathbf{x}}\left(\left(L_{\mathbf{v}}^{s}\right)^{T}\right)=\frac{\partial}{\partial \mathbf{x}}\left(\mathbf{S}_{\mathbf{v}}^{f T} a+\mathbf{S}_{\mathbf{v}}^{t T} a\right)=\left.\mathbf{S}_{\mathbf{v x}}^{f}\right|_{\lambda=a}+\left.\mathbf{S}_{\mathbf{v x}}^{t}\right|_{\lambda=a}
\end{aligned}
$$

In this paper, we only minimize the active power loss. Then, the gradient and Hessian matrix of the real power loss can be simply obtained by taking the real part of that of the complex power loss. Note that matrix blocks in the gradient and Hessian matrix of generation costs related to line reactance are zero matrices, and the remaining matrix blocks are identical to the results in [11].

\section{CASE STUdY}

To validate the validity of the proposed ACOPF model and effectiveness of the developed interior-point solver, we conduct case studies on the IEEE 118-bus transmission system. The algorithms are performed on a laptop with Intel Core i7 processor CPU $2.90 \mathrm{GHz}$ with 8 GB RAM.

We compare generation costs, system losses, and CPU time in the following three cases. Case 0: the conventional ACOPF is applied; Case 1: the proposed ACOPF model with $\omega_{1}=0$ and $\omega_{2}=1$ is used only to minimize the generation cost; Case 2: the proposed ACOPF model with $\omega_{1}=1000$ and $\omega_{2}=1$ is used to minimize the generation cost and the system loss. We identify the maximum line power flow using the conventional ACOPF under the default load in MATPOWER, denoted by $S_{\max }^{f}$. Then, we make the power flow limit of each line equal to $k \times S_{\max }^{f}$, where factor $k=\{0.4,0.6,0.8,1\}$ in different tests. We assume that D-FACTS devices are installed on all lines, and set $\eta=20 \%$ to be consistent with the D-FACTS setting in [6].

The simulation results are listed in Table I. The generation cost in the proposed ACOPF is always less than that in the conventional ACOPF since the dispatchable line reactance can reduce the congestion in the system. The system loss in Case 2 is always less than that in Cases 0 and 1 under different flow limit conditions, which indicates the effectiveness of the proposed ACOPF model in minimizing system losses. The CPU time for solving the proposed ACOPF is less than 15 seconds in most cases, suggesting that the proposed ACOPF model can be applied in real-time system operations using the modified interior-point solver.

TABLE I

COSTS, LOSSES AND CPU TIME UNDER DIFFERENT FLOW LIMIT CONDITIONS

\begin{tabular}{|c|c|c|c|c|c|}
\hline$k$ & & 0.4 & 0.6 & 0.8 & 1.0 \\
\hline \multirow{3}{*}{$\begin{array}{c}\text { Generatio } \\
\mathrm{n} \\
\text { Cost }(\$)\end{array}$} & Case 0 & 131,395 & 130,337 & 129,830 & 129,660 \\
\cline { 2 - 6 } & Case 1 & 131,219 & 130,150 & 129,643 & 129,475 \\
\cline { 2 - 6 } System & Case 2 & 131,242 & 130,170 & 129,664 & 129,498 \\
\cline { 2 - 6 } $\begin{array}{c}\text { Loss } \\
(\mathrm{MW})\end{array}$ & Case 0 & 67.33 & 70.73 & 73.54 & 77.39 \\
\cline { 2 - 6 } & Case 1 & 65.45 & 67.09 & 70.25 & 74.07 \\
\hline \multirow{2}{\text{CPU}}{$\begin{array}{c}\text { Time }(\mathrm{s}) \\
\end{array}$} & Case 0 & 60.63 & 63.04 & 65.91 & 69.18 \\
\cline { 2 - 6 } & Case 1 & 5.58 & 3.93 & 2.41 & 0.45 \\
\cline { 2 - 6 } & Case 2 & 4.81 & 5.66 & 4.64 & 13.24 \\
\hline
\end{tabular}


To further verify the effectiveness of the proposed ACOPF model, we investigate the impact of D-FACTS placement on system losses. We use power loss to impedance sensitivity (PLIS) to determine the most appropriate D-FACTS locations for minimizing system losses, which is a linearized weight of the transmission line to indicate a system loss change due to a change in the line impedance [12]. Simulations are also carried out in the IEEE 118-bus system under heavy load conditions. To focus on studying the system loss, we set $\omega_{1}=1$ and $\omega_{2}=0$. We construct the following five D-FACTS placement cases, in which we install D-FACTS devices on 62 out of 179 lines.

Case 0: This is the base case where no D-FACTS devices are used in the system.

Case 1: D-FACTS devices are installed on 62 lines with the lowest PLIS.

Case 2: D-FACTS devices are placed on 62 randomly selected lines.

Case 3: D-FACTS devices are installed on 62 lines with the highest PLIS.

Case 4: D-FACTS devices are installed on all 179 lines in the system.

The total PLIS of the lines equipped with D-FACTS devices, system losses, and the loss reduction in the above five cases are summarized in Table II. Installing D-FACTS devices on the lines with the highest PLIS values in Case 3 can reduce $1.52 \%$ of system loss compared with that in Case 0 , while installing D-FACTS devices on the lines with the lowest PLIS values can only reduce $0.25 \%$ of system loss compared with that in Case 0. The comparison in Table II illustrates that the loss reduction increases with the increase in PLIS values, which is consistent with the conclusion in [12]. The simulation results verify the effectiveness of the proposed ACOPF model and the modified interior-point solver.

TABLE II

SYSTEM LOSSES UNDER DIFFERENT D-FACTS PLACEMENTS

\begin{tabular}{|c|c|c|c|}
\hline $\begin{array}{c}\text { D-FACTS } \\
\text { placement }\end{array}$ & $\begin{array}{c}\text { PLIS } \\
\text { sum }\end{array}$ & $\begin{array}{c}\text { Loss } \\
(\mathrm{MW})\end{array}$ & $\begin{array}{c}\text { Decrease } \\
(\%)\end{array}$ \\
\hline Case 0 & 0 & 30.67 & -- \\
\hline Case 1 & 0.10 & 30.59 & 0.25 \\
\hline Case 2 & 1.55 & 30.55 & 0.38 \\
\hline Case 3 & 8.55 & 30.21 & 1.52 \\
\hline Case 4 & 9.19 & 30.08 & 1.93 \\
\hline
\end{tabular}

\section{CONCLUSION}

This paper proposes an ACOPF model considering the DFACTS devices, in which the reactance of lines equipped with D-FACTS devices are introduced as the decision variables. The proposed ACOPF model can be seamlessly integrated into the existing energy management system of a power system in the control room. System operators can apply the proposed ACOPF model to manage the real-time system operation and determine the setpoints of D-FACTS devices. Furthermore, the setpoints of D-FACTS devices can be adopted by MTD to safeguard a cyber-secure power system. In addition, we derive the gradient and Hessian matrices of the objective function and constraints with respect to the line reactance, which are used to build an interior-point solver of the proposed ACOPF. Since the derivations in this paper adopt the same voltage coordinate and complex power expression as MATPOWER, the derived gradient and Hessian matrices can be simply integrated into MATPOWER. The case study compares the proposed ACOPF with the conventional ACOPF regarding generation costs, system losses and CPU time under different power flow limits. The results show that D-FACTS devices can effectively reduce the system loss, and CPU time of solving the proposed ACOPF is generally less than $15 \mathrm{~s}$. The case studies also investigate the impact of D-FACTS placement on system loss. The results verify the effectiveness of the proposed ACOPF model and the interior-point solver.

In future work, we will utilize the proposed model in MTD to detect cyber-attacks in the transmission system. We will investigate the impact of the setpoints of D-FACTS devices on the detection effectiveness of MTD.

\section{ACKNOWLEDGMENT}

This material is based upon work supported by the Department of Energy, Office of Energy Efficiency and Renewable Energy (EERE), Solar Energy Technologies Office, under Award Number DE-EE0008767.

\section{REFERENCES}

[1] B. Li, and X. Li, "Distributed Generation Sources and Their Effects on Distribution Networks," International Electric Power For China, vol. 9, no. 3, pp. 46-49, June. 2005

[2] J. Wang, Z. Wang, L. Xu, and Z. Wang, "A Summary of Applications of D-FACTS on Microgrid," in 2012 Asia-Pacific Power and Energy Engineering Conference, 2012, pp. 1-6.

[3] D. K. Dhaked and M. Lalwani, "A Review Paper on A DFACTS Controller: Enhanced Power Flow Controller," vol. 10, no. 1, p. 9 .

[4] B. Liu, H. Wu, A. Pahwa, F. Ding, E. Ibrahim, and T. Liu, "Hidden Moving Target Defense against False Data Injection in Distribution Network Reconfiguration," in 2018 IEEE Power Energy Society General Meeting (PESGM), 2018, pp. 1-5.

[5] B. Liu, and H. Wu, "Optimal D-FACTS Placement in Moving Target Defense against False Data Injection Attacks," in IEEE Transactions on Smart Grid.

[6] C. Liu, J. Wu, C. Long, and D. Kundur, "Reactance Perturbation for Detecting and Identifying FDI Attacks in Power System State Estimation," IEEE J. Sel. Top. Signal Process., vol. 12, no. 4, pp. 763-776, Aug. 2018.

[7] O. A. Urquidez, and L. Xie, "Rectangular Representation of FACTS Devices in the ACOPF Problem," in 2014 North American Power Symposium (NAPS), Pullman, WA, USA, 2014, pp. 1-6.

[8] T. C. Subramanyam, J. B. Subrahmanyam, and T. Ram, "An Adaptive Chicken Swarm Algorithm to Solve Optimal Power Flow Problem Considering FACTS Device," Journal of Computational Mechanics, Power Syst. and Control, vol. 2, no. 4, pp. 38-47, Oct. 2019.

[9] G. L. Torres and V. H. Quintana, "An Interior-point Method for Nonlinear Optimal Power Flow using Voltage Rectangular Coordinates," IEEE Trans. Power Syst., vol. 13, no. 4, pp. 1211-1218, Nov. 1998.

[10] G. L. Torres, and M. A. De Carvalho, "On Efficient Implementation of Interior-Point Based Optimal Power Flows in Rectangular Coordinates," in 2006 IEEE PES Power Systems Conference and Exposition, 2006, pp. 1747-1752.

[11] R. D. Zimmerman, "AC Power Flows, Generalized OPF Costs and their Derivatives using Complex Matrix Notation." Cornell University, 2008.

[12] K. M. Rogers, and T. J. Overbye, "Some Applications of Distributed Flexible AC Transmission System (D-FACTS) Devices in Power Systems," in 2008 40th North American Power Symposium, 2008, pp. 1-8. 